\title{
Sensitivity problems of control system of coal preparation processes
}

\author{
Roman Kaula ${ }^{1, a}$ \\ ${ }^{1}$ Silesian University of Technology, Department of Electrical Engineering and Control in Mining, 44-100 Gliwice, \\ Akademicka 2, Poland
}

\begin{abstract}
Control of technological processes of coal preparation takes place in the presence of wide disturbances. An important problem is the choice of the controller which is robust for a variety of disturbances. No less important problem in the control process is the tuning of the controller parameters. In the paper the analysis of influence of changes in object model parameters on the course of the controlled value was carried out. For the controller settings, calculated according to model parameters research was carried out on object with other values of parameters. In the studies a sensitivity analysis method was used. The sensitivity analysis for the three methods of tuning PI controller for control systems of coal preparation processes characterized by dynamic properties of the inertial element with time delay was presented. Considerations were performed at various parameters of the object on the basis of the response of the control system for a constant value of the set point. The assessment of the considered tuning methods based on selected indices of control quality was realized.

One of the primary tasks of the control system is to maintain the value of the controlled variable (process output) at the desired level. An important problem from the point of view of the control quality is also getting the appropriate course of the controlled variable and reduce the influence of disturbances running on the system. Compliance with these requirements may be accomplished by an appropriate selection of the structure of the controller and its settings. Many methods of tuning the controller use a dynamic model of a control object (determined on the basis of dynamic characteristics of the controlled process). The description of the properties of the dynamic process by using a mathematical model is helpful in the design of the control system. Knowing the dynamic model and its parameters we can analytically determine the controller settings for the assumed criterion of the control quality. Different processes with the same dynamic properties can be described by a dynamic model of the same structure which differs in only parameter values. Such an approach makes it possible to apply the same type of controller in control systems of various industrial processes. This also applies to the processes of coal preparation.

The dynamic properties of many processes of coal preparation can be characterized by the inertial model of the first order (described time constant $T$ ) with time delay $\tau$ [1-4].

The PI controllers have two tuning parameters to adjust: the proportional gain $\left(k_{r}\right)$ and integral time $\left(T_{i}\right)$. One of the basic methods of tuning of the PI controller is the method of Ziegler-Nichols of the identification test [5]. The Z-N method may be the initial method of tuning for many processes of the coal preparation where the dynamics has the properties of the
\end{abstract}

${ }^{\text {a } C o r r e s p o n d i n g ~ a u t h o r: ~ r k a u l a @ p o l s l . p l ~}$ 
inertial system of the first order with time delay. Many authors dealing with the tuning of PI controllers parameters for processes with dynamics of the first order inertial model proposes settings based on the reduction of the time constant [6]. In these methods (R) it is assumed that the parameter $T_{i}$ of the integral term is equal to the time constant $T_{i}=T$ of the system. Differences in the settings are the result of the adopted tuning criteria of the parameter $k_{r}$. In the paper [7] a new approach for tuning PI controller for inertial object with time delay has been proposed. In the direct method (D) of controller tuning an additional criterion was used. The condition on the phase margin from the Nyquist criterion was applied.

It should be noted that the identification of the object parameters (including the time constant) under industrial conditions is usually performed during normal operation (with the influence of disturbances). Thus, determined parameters of the dynamic model may be different from the actual values of the process. The control system with controller which is tuned on the basis of such the model may not satisfied assumed requirements of the control quality. In the paper we analyse the impact of changes of the object model parameters on the course of the controlled variable. In the study the sensitivity analysis has been used.

One of the important indicators to assess the control quality is a parameter describing the sensitivity of the controlled variable on change in the object parameters. Changes of two parameters $\alpha=\{T, \tau\}$ for the object were analyzed. The controlled variable in the Laplace form $\mathrm{Y}(\mathrm{s})$ can be written:

$$
Y(s)=K_{c l}(s) Y_{r e f}(s)
$$

where: $\mathrm{Y}_{\text {ref }}(\mathrm{s})$ - reference value of the controlled object,

$\mathrm{K}_{c l}(\mathrm{~s})$ - closed loop transfer function.

Thus, the changes of the controlled variable and the changes of the model parameter $\alpha=\{T, \tau\}$ are as follows:

$$
Y(s, \alpha)+\Delta Y(s, \alpha)=K_{c l}(s, \alpha) Y_{r e f}(s)+\Delta K_{c l}(s, \alpha) Y_{r e f}(s)
$$

Relative sensitivity of the transfer function of the closed system according to parameter $\alpha=\{T, \tau\}$ can be expressed by the formula:

$$
S_{K c l(\alpha)}=\frac{\frac{\Delta K_{c l}(s, \alpha)}{K_{c l}(s, \alpha)}}{\frac{\Delta \alpha}{\alpha}}=\frac{\alpha}{K_{c l}(s, \alpha)} \frac{\partial K_{c l}(s, \alpha)}{\partial \alpha} .
$$

After transformations:

$$
\Delta K_{c l}(s, \alpha)=\frac{K_{R}(s)}{\left(1+K(s, \alpha) K_{R}(s)\right)^{2}} \Delta K(s, \alpha)
$$

where: $\mathrm{K}(\mathrm{s}, \alpha), \mathrm{K}_{\mathrm{R}}(\mathrm{s})$ - the transfer function of object and controller,

$$
\begin{aligned}
& \Delta K\left(s, \alpha_{T}\right)=\frac{k}{T_{2} s+1} e^{-\tau s}-\frac{k}{T_{1} s+1} e^{-\tau s} \\
& \Delta K\left(s, \alpha_{\tau}\right)=\frac{k}{T s+1} e^{-\tau_{2} s}-\frac{k}{T s+1} e^{-\tau_{1} s},
\end{aligned}
$$

$T_{1}, \tau_{l}$ - parameter values for which the controller parameters have been determined,

$T_{2}, \tau_{2}-$ actual values of the system parameters. 
The paper presents the sensitivity analysis for the three methods of tuning PI controller. Based on the dependences presents in works [5-7] the values of the PI controller parameter settings (depending on the parameters $k, T, \tau$ of the dynamics model) have been calculated.

Data of the parameters of the dynamic model from work [3] were taken. In this work the dynamic model parameters of the coal flotation process using various methods of identification were determined. Depending on the chosen method of identification and the set point of the system, different values of system parameters (time constant $T$ and the delay $\tau$ ) were obtained. The time constant $T$ in the range of values (200-800) s, while the time delay $\tau$ in the range of values (200-400) s have been identified. It is therefore advisable to analyze the control system for the process with parameters that differ even tens of percent from parameters used to controller settings.

Table 1. Indices of the control quality.

\begin{tabular}{|c|c|c|c|c|c|c|c|c|c|}
\hline \multirow[t]{2}{*}{ no } & \multirow[t]{2}{*}{$\Delta \mathrm{T}$} & \multirow[t]{2}{*}{$\Delta \tau$} & \multirow[t]{2}{*}{$\frac{T \pm \Delta T}{\tau \pm \Delta \tau}$} & \multicolumn{3}{|c|}{$\begin{array}{c}\begin{array}{c}\text { Integral criterion } \\
\text { in the relative } \\
\text { terms }\end{array} \\
\frac{I_{2}}{I_{2 \min }} 100 \\
, \%\end{array}$} & \multicolumn{3}{|c|}{$\begin{array}{c}\text { Rise time } t_{n}, s \\
\left.\text { (Settling time } t_{u}, s\right)\end{array}$} \\
\hline & & & & $\mathrm{ZN}$ & $\mathrm{R}$ & D & $\mathrm{ZN}$ & $\mathrm{R}$ & D \\
\hline 1 & $+0.5 \mathrm{~T}$ & 0 & 3 & 190 & 295 & 237 & $\begin{array}{c}343 \\
(1200)\end{array}$ & $\begin{array}{c}1015 \\
(2861)\end{array}$ & $\begin{array}{c}557 \\
(2400)\end{array}$ \\
\hline 2 & $-0.5 \mathrm{~T}$ & 0 & 1 & 267 & 208 & 170 & $\begin{array}{c}136 \\
(5200)\end{array}$ & $\begin{array}{c}1324 \\
(2100)\end{array}$ & $\begin{array}{c}667 \\
(1378) \\
\end{array}$ \\
\hline 3 & 0 & $+0.5 \tau$ & $4 / 3$ & 318 & 303 & 278 & $\begin{array}{c}214 \\
(4800)\end{array}$ & $\begin{array}{c}686 \\
(2640)\end{array}$ & $\begin{array}{c}400 \\
(2520)\end{array}$ \\
\hline 4 & 0 & $-0.5 \tau$ & 4 & 117 & 211 & 151 & $\begin{array}{c}786 \\
(1757)\end{array}$ & $\begin{array}{c}1324 \\
(1862)\end{array}$ & $\begin{array}{c}760 \\
(1120)\end{array}$ \\
\hline 5 & $+0.5 \mathrm{~T}$ & $+0.5 \tau$ & 2 & 278 & 352 & 316 & $\begin{array}{c}331 \\
(3062)\end{array}$ & $\begin{array}{c}920 \\
(3280)\end{array}$ & $\begin{array}{c}480 \\
(2400)\end{array}$ \\
\hline 6 & $+0.5 \mathrm{~T}$ & $-0.5 \tau$ & 6 & 139 & 250 & 184 & $\begin{array}{c}643 \\
(1114)\end{array}$ & $\begin{array}{c}1311 \\
(1714)\end{array}$ & $\begin{array}{c}827 \\
(2193)\end{array}$ \\
\hline 7 & $-0.5 \mathrm{~T}$ & $+0.5 \tau$ & $2 / 3$ & $\begin{array}{l}\text { unst } \\
\text { able }\end{array}$ & 257 & 271 & - & $\begin{array}{c}386 \\
(2143)\end{array}$ & $\begin{array}{c}240 \\
(3680)\end{array}$ \\
\hline 8 & $-0.5 \mathrm{~T}$ & $-0.5 \tau$ & 2 & 100 & 171 & 119 & $\begin{array}{c}124 \\
(1945)\end{array}$ & $\begin{array}{c}1600 \\
(2400)\end{array}$ & $\begin{array}{c}1000 \\
(1680)\end{array}$ \\
\hline
\end{tabular}

The simulation studies of the response of the control system on step change of the set point were carried out. In each considered case a constant value of gain $k=1$ of the object was assumed. The controller settings for $T=500 \mathrm{~s}$ and $\tau=250 \mathrm{~s}$ were calculated. The study on the system with parameters $T \pm 0,5 T$ and $\tau \pm 0,5 \tau$ was carried out. Changes in one and both parameters simultaneously were assumed.

The results are shown in Table 1. To assess the control quality, the criterion of the integral of the squared control error (in relative terms) and parameters: rise time $t_{n}$ and settling time $t_{u}$ were used.

The sensitivity analysis for three methods of the tuning PI controller in the control systems of coal preparation processes characterized by the dynamic properties of the inertial system with time delay was carried out. The studies of the control system at the object parameters which differed to a certain range from $T$ and $\tau$ (Table 1) were performed.

Responses of the control system for a step change in the set point were obtained. Based on the courses it can be noted that for all considered cases (except one) regardless of the choice of the method of the parameter settings of the PI controller, the controlled variable assumes a steady state value. This shows a large robustness of the PI controller on disturbances 
connected with the change of the dynamic properties of the object (or inaccurate identification of the parameters). Only at controller settings according to the $\mathrm{ZN}$ criterion for case 7 the system is unstable. In this case the ratio $((T-\Delta T) /(\tau-\Delta \tau))=2 / 3$ is less than the ratio $T / \tau=2$ for which the settings of the PI controller were tuned. The situation in which the ratio of ( $T$ $\Delta T) /(\tau-\Delta \tau))$ is less than the $T / \tau$ ratio refers also to cases 2 and 3. In these cases we may notice the presence of the larger oscillations of the controlled variable.

The best results of the control (the control quality) from the point of view of the integral index for cases 4 and 8 were obtained. This occurs when the parameter of the time delay $\tau$ (accepted for the settings) is larger than the actual time delay of the object. Analyzing the transient nature of the controlled variable and the quality parameters it should be noted that in these cases were achieved better control quality than at the control system in which the controller settings for the actual object parameters were determined. Comparing indices of the control quality for the analyzed methods of the tuning of the controller settings you will notice the largest variability for the $\mathrm{ZN}$ method and the smallest for the method of reduction (R). The transients of the controlled variable for the tuning method of $\mathrm{ZN}$ in most cases are oscillatory. The oscillations are not present in the transients of the controlled variable for the method of the reduction $(\mathrm{R})$. This is achieved at the expense of significant deterioration of other quality parameters. The transients have the longest settling time and rise time for the settings according to the criterion of the reduction (R). The value of the integral index is the smallest in five cases for the method of $\mathrm{ZN}$ and in two cases for the direct method (D). In most cases the shortest settling time of the transient in the direct method (D) was obtained.

For the assumption of larger time delay in the controller settings, relative to the real delay of the system (identified parameter larger than actual), the quality of control was improved. The obtained results in identifying the parameters of the system model and a new approach to the tuning of the PI controller can be very helpful.

\section{References}

1. S. Cierpisz, in Proceedings of IX Konferenca Automatyzacji Procesów Przeróbki Kopalin, (Szczyrk, 49, 2003)

2. S. Cierpisz, Monograph, Wydawnictwo Politechniki Śląskiej, Gliwice, 403 (2012)

3. J. Joostberens, MiAG, 12, 34 (2011)

4. K. Kalinowski K., R. Kaula, MiAG, 1, 35 (2000)

5. J. Ziegler, N. Nichols, Trans. ASME, 64, 759 (1942)

6. A. O'Dwyer, Handbook of PI and PID controller tuning rules, Imperial College Press, London (2003)

7. R. Kaula, GSM-MRM, 31(1), 141 (2015) 